\title{
RIGHLIGHTS
}

PROSTATE CANCER

\section{Individualized treatment recommended in older men}

The International Society of Geriatric Oncology (SIOG) has published its recommendations for the management of prostate cancer in men aged $\geq 70$ yearsthe age group that is predominantly affected by this disease. Based on a systematic search of the published literature on screening, diagnostic procedures and treatment options for both localized and advanced prostate cancer, the SIOG Prostate Cancer Task Force has concluded that older men with prostate cancer should be treated on the basis of their state of health, and not solely according to their age.

The task force members believe that the decision on how to treat prostate cancer should follow internationally accepted treatment guidelines, such as those published by the European Association of Urology, but should also be based on evaluation of the individual patient's health status and prognostic factors. Particularly important is assessment of comorbidities (using the Cumulative Illness Score RatingGeriatrics [CISR-G] scale), patient independence (using the Instrumental Activities of Daily Living [IADL] and ADL scales), and nutritional status (using weight-loss estimation). Patients could then be categorized into four main groups and treatment tailored accordingly.

'Healthy' men (independent and well-nourished with controlled comorbidities) should be given the same treatment options as younger patients. 'Vulnerable' men (some dependency or risk of malnutrition, and reversible impairment) should be offered standard therapies once their impairment is resolved. Treatment of 'frail' men (irreversible impairment, highly dependent, severe malnutrition) should be adapted to focus on symptom management. 'Terminal' patients (bedridden, major comorbidities, cognitive impairment) should receive palliative care only. Decision trees for both localized prostate cancer (outlined in the above example) and metastatic disease are published in the task force's recent BJU International paper.

Existing guidelines for the management of prostate cancer do not provide specific recommendations for the treatment of older men. The SIOG task force members argue that the risks and benefits of treatment should be assessed on an individual basis, so as not to decrease quality of life without improving survival. "We believe that an important objective of clinical research ... should be to improve this profiling process ... to predict outcome and to decide upon geriatric and oncologic interventions" concludes lead author Jean-Pierre Droz.

Rebecca Drake

Original article Droz, J.-P. et al. Management of prostate cancer in older men: recommendations of a working group of the International Society of Geriatric Oncology. BJU Int. doi:10.1111/j.1464-410X.2010.09334.x 